population in 1985-2005 and to quantify the contribution of variation in population's size and age structure, and risk to the change in number of deaths.

Methods The number of deaths from CVD (CID10: I00-199) and all malignant tumours (CID10: C00-C99) and the population, by sex and age, were obtained from official statistics. We standardised mortality rates (direct method, European population) and used joinpoint analysis to identify changes in trends and to estimate the annual percent change (APC) of the standardised rate. We used the tool RiskDiff to quantify the contribution of risk, size and structure of the population during the periods with constant log-linear trend.

Results Mortality from CVD declined since 1985, most sharply since 1993 (men: $\mathrm{APC}=-4.0 \%, 95 \% \mathrm{CI}-3.5$ to -4.6 ; women: $\mathrm{APC}=-3.8 \%, 95 \% \mathrm{CI}-3.1$ to -4.4$)$. The increasing population and its ageing explain a $30 \%$ increase in the number of deaths. The risk reduction explains a reduction by half, resulting in fewer deaths from CVD (men: $-24 \%$, women: $-17 \%)$. Mortality from malignant tumours significantly increased until 1996 in men (APC $=0.8 \%, 95 \%$ CI 0.5 to 1.1). Since 1996 in men and 1991 in women, despite the growing number of deaths (men: $13 \%$, women: $16 \%$ ), the risk is declining as illustrated by decreasing standardised mortality rates (men: $\mathrm{APC}=-0.4 \%, 95 \% \mathrm{CI}-0.7$ to -0.1 ; women: $\mathrm{APC}=-0.8 \%$, 95\% CI -0.9 to -0.6$)$.

Conclusion In Portugal, the risk of death from CVD and cancer is decreasing, although this is reflected in fewer deaths only for CVD.

\section{P2-241 EVALUATION OF THE NATIONAL CERVICAL SCREENING PROGRAMME IN CHILE}

doi:10.1136/jech.2011.142976j.74

${ }^{1}$ A Pereira, * ${ }^{2}$ Silva, ${ }^{2} J$ Peto. ${ }^{1}$ School of Public Health, University of Chile, Santiago, Chile; ${ }^{2}$ London School of Hygiene and Tropical Medicine, London, UK

Introduction The Chilean Cervical Screening Programme (CCSP) was implemented nationally in 1994, but its performance and effectiveness in reducing mortality from cervical cancer (CC) have not been assessed at an individual level. This is the first comprehensive evaluation of a national screening programme in a developing country based on individual record-linkage.

Methods We linked three Chilean national datasets using the unique personal identification number: (1) the National Health Service (NHS) dataset, which holds data on all women registered in the public health sector; (2) the Cito-Expert dataset, which contains all NHS screening records; and (3) national data on underlying causes of all deaths. This linkage provided a retrospective cohort of 2.8 million women aged 25-64 years followed from 1997 to 2007. The CCSP was assessed in terms of its performance and effectiveness in reducing CC mortality.

Results About $30 \%$ of the women in the cohort were never screened during the study period in the public health sector. The 5-year coverage was $\sim 60 \%$, with over-screening in specific subgroups, and $\sim 60-70 \%$ of LSIL+ smears had three following normal smears within 48 months. Women ever screened had a 30\% reduction in CC mortality relative to those who had never been screened (adjusted rate ratio: $0.7,95 \% \mathrm{CI} 0.6$ to 0.8 ). The effectiveness of the programme was higher in middle-aged women ( $35-54$ years) and in the lowest socioeconomic group.

Discussion The CCSP has reduced CC mortality during the last decade, but its impact could be increased by improving coverage and follow-up of abnormal smears.

\section{P2-242 TOOTH LOSS IS ASSOCIATED WITH INCREASED BLOOD PRESSURE IN ADULTS: A BRAZILIAN POPULATION-BASED STUDY}

doi:10.1136/jech.2011.142976j.75

${ }^{1} \mathrm{M}$ Peres, ${ }^{*}{ }^{2} \mathrm{G}$ Tsakos, ${ }^{1} \mathrm{P}$ Barbatto, ${ }^{3} \mathrm{D}$ Silva, ${ }^{1} \mathrm{~K}$ Peres. ${ }^{1}$ Post-Graduate Program in Public Health, Federal University of Santa Catarina, Florianopolis, Santa Catarina, Brazil; ' 2 Department of Epidemiology and Public Health, University College London, London, UK; ${ }^{3}$ Post-Graduate Program in Physical Education, Federal University of Santa Catarina, Florianopolis, Santa Catarina, Brazil

Introduction In addition to well-known risk factors, markers of dental disease and inflammation, such as tooth loss, are suggested to be also independently associated with blood pressure.

Objectives To investigate whether tooth loss is associated with increased blood pressure among adults, independently of the established risk factors.

Methods A population-based cross-sectional study was carried out with a sample of 1720 adults from Florianópolis, Brazil. Data collection included blood pressure, anthropometric measures and a questionnaire on socio-demographics, self-rated health, diabetes, self-reported number of natural teeth and dental prosthesis. We used multivariable regression models for the association between systolic blood pressure (SBP), diastolic blood pressure (DBP) and high levels of blood pressure (HBP) and tooth loss, sequentially adjusting for socio-demographic, behavioural and health related confounders.

Results Adjusted analysis revealed a Prevalence Ratio of HBP of 1.42 (95\% CI 1.15 to 1.75 ) and 1.06 (95\% CI 0.91 to 1.24 ) for edentate adults and adults with $<10$ teeth in at least one arch respectively, compared to those with 10 or more teeth in both arches. An association between edentate men and SBP was observed in the unadjusted but not the adjusted analysis. However, among women, edentate participants showed significantly higher SBP when compared with those with 10 or more teeth in both arches, after adjusting for potential confounders.

Conclusions There is an association between tooth loss and increased blood pressure and high levels of blood pressure in the general adult population of Florianopolis, especially among women, in addition to well-known hypertension risk factors.

\section{P2-243 THE PROTECTIVE EFFECT OF PREDOMINANT AND EXCLUSIVE BREASTFEEDING ON MALOCCLUSION: RESULTS FROM A BRAZILIAN BIRTH COHORT}

doi:10.1136/jech.2011.142976j.76

${ }^{1} \mathrm{~K}$ Peres, ${ }^{* 1} \mathrm{M}$ Peres, ${ }^{2} \mathrm{~A}$ Cascaes, ${ }^{2} \mathrm{~F}$ Demarco, ${ }^{2} \mathrm{~A}$ Matijasevich, ${ }^{2} \mathrm{~S}$ Santos, ${ }^{2} \mathrm{~A}$ Barros. ${ }^{1}$ Universidade Federal de Santa Catarina, Florianópolis, Brazil; ${ }^{2}$ Universidade Federal de Pelotas, Pelotas, Brazil

Introduction There is a lack of evidence supporting the protective effect of breastfeeding on occlusion in primary dentition. The aim of this study was to examine the effects of predominant $(\mathrm{PB})$ and exclusive breastfeeding (EB) on malocclusion adjusting for recognised risk factors.

Methods A cross-sectional study was carried out nested in a population-based birth cohort from Pelotas, Brazil which started in 2004 A sample of 1129 children aged 5 years old underwent dental examination. Anterior open bite, posterior cross bite, canines relationship, and overjet, were recorded according (1) Foster- Hamilton criteria and (2) WHO criteria. A history of breastfeeding and nonnutritive sucking habits was collected at interview with the child's mother. Data were analysed using multivariable Poisson regression controlling for potential confounders.

Results The mean duration of $\mathrm{EB}$ and $\mathrm{PB}$ were higher among children free of malocclusions; the longer the duration of breastfeeding, 
the lower the prevalence of malocclusions. The concomitant presence of $\mathrm{PB}$ for at least six months and the non-regular use of pacifier until two years of age show a protective effect for crossbite following adjustment for confounders. The longer the duration of $\mathrm{EB}$ the lower the prevalence of moderate or severe WHO malocclusion. A dose-response relationship between duration of $\mathrm{EB}$ or $\mathrm{PB}$ and the prevalence of malocclusion was observed. This was reduced or eliminated if pacifier use is introduced early in the child's life. Conclusions Given that breastfeeding is a protective factor for several childhood diseases, our findings indicate that the common risks approach is the most appropriate way for the prevention of malocclusions

\section{P2-244 APOLIPOPROTEIN E GENOTYPE, LIPOPROTEINS AND ISCHAEMIC HEART DISEASE IN LITHUANIAN POPULATION}

\section{doi:10.1136/jech.2011.142976j.77}

J Petkeviciene,* A Smalinskiene, D Luksiene, K Jureniene, A Tamosiunas, $\mathrm{J}$ Klumbiene, V Lesauskaite. Medical Academy of Lithuanian University of Health Sciences, Kaunas, Lithuania

Introduction The aim of the study was to assess the frequency of APOE genotypes and their relationship with serum lipoproteins and prevalence of ischaemic heart disease (IHD) in Lithuanian population. Methods Cross- sectional health survey was carried out in representative random samples of Lithuanian urban and rural population (936 men and 1115 women) aged 25-70. Serum low-density lipoprotein cholesterol (LDL-C) was determined by enzymatic methods. Three APOE genotypes were identified by polymerase chain reaction: $A P O E 2$ for those subjects carrying $\epsilon 2 / 2$ or $\epsilon 2 / 3$ genotypes, $A P O E 3$ - for $\epsilon 3 / 3$ genotype, and APOE 4 for - $\epsilon 3 / 4$ or $\epsilon 4 / 4$ genotypes. IHD was diagnosed according to the data of medical history and medical records, Rose questionnaire and by the Minnesota codes of electrocardiogram. Multiple logistic regression analysis was used to explore the impact of $A P O E$ genotype on risk of IHD.

Results The frequencies of $A P O E$ genotypes did not differ between men and women. The observed frequency of APOE3 genotype was the highest (63\%). APOE2 genotype and APOE4 genotype were found in $17 \%$ and $17.2 \%$ of population respectively. In both genders individuals with APOE2 genotype had the lowest level of LDL-C. After adjustment for age and other conventional IHD risk factors, the OR for myocardial infarction for APOE2 men vs APOE3 men was 0.12 (95\% CI 0.02 to 0.84$)$. No relationship was found between $A P O E$ genotypes and IHD in women.

Conclusion This study suggests that $A P O E$ genotype influences the level of plasma lipoproteins in both gender and risk of IHD in men.

\section{P2-245 PREVALENCE OF HYPERTENSION IN BRAZIL OVER THE PAST 3 DECADES: A SYSTEMATIC REVIEW AND META- ANALYSIS}

doi:10.1136/jech.2011.142976j.78

${ }^{1} \mathrm{R}$ V Picon, ${ }^{*}{ }^{1} \mathrm{G}$ Riegel, ${ }^{1,2} \mathrm{~L}$ B Moreira, ${ }^{1,2} \mathrm{~F}$ D Fuchs, ${ }^{1,2} \mathrm{~S}$ C Fuchs. ${ }^{1}$ Universidade Federal do Rio Grande do Sul, Porto Alegre, Rio Grande do Sul, Brazil; ${ }^{2}$ Hospital de Clínicas de Porto Alegre, Porto Alegre, Rio Grande do Sul, Brazil

Introduction Hypertension has become a major public health concern in developing countries. We conducted a systematic review with meta-analysis of population-based studies in order to estimate the prevalence of hypertension among the Brazilian adult population. Methods Published cross-sectional and cohort studies conducted from 1980 to 2010 were independently identified by two reviewers, without language restriction, in the PubMed, Embase, LILACS, and SciELO electronic databases. Unpublished studies were explored in the CAPES electronic thesis database. Hypertension was defined by JNC criteria of 140/90 mm Hg blood pressure measurement or use of blood pressure lowering medication, and additionally selfreported hypertension.

Results The search retrieved 598 original studies, 23 thesis, and six references from manual search. Based in title and abstract, 450 articles were excluded and 60 in full-text screening, leaving 47 eligible studies. Further five results were excluded, based on sampling and biases, and 42 studies comprising 124 thousand individuals were included. In the 1980's ( $\mathrm{n}=5)$, pooled JNC prevalence of hypertension was $35.9 \%$ (95\% CI $28.4 \%$ to $44.2 \%$ ), $45.1 \%$ ( $40.0 \%$ to $50.4 \%)$ among men, and $34.6 \%$ (23.7\% to $47.5 \%)$ among women. In the 1990's $(n=15)$, the prevalence rates were, respectively, $28.5 \%$ (21.4\% to $36.9 \%$ ), $24.6 \%$ (15.5\% to $36.6 \%$ ), and $23.0 \%$ ( $14.5 \%$ to $34.3 \%)$. In the 2000 's $(n=22)$, the prevalence rates were $29.6 \%$ (26.3\% to $32.9 \%$ ); $29.4 \%$ (24.1\% to $-35.3 \%$ ), and $25.8 \%$ (20.5\% to $31.9 \%$ ). Pooled prevalence from self-reported hypertension on telephone enquires, the overall was $20.6 \%$ (19.0\% to $22.4 \%), 18.6 \%$ $(17.4 \%$ to $19.9 \%)$ in men and $23.2 \%(21.1 \%$ to $25.4 \%)$ in women.

Conclusions This meta-analysis was first to summarise prevalence rates for Brazil. Steady estimates were detected over 3 decades, although lower rates for self-reported hypertension.

\section{P2-246 THE NIGHTINGALE STUDY: A PROSPECTIVE COHORT STUDY ON SHIFT WORK AND BREAST CANCER AMONG NURSES IN THE NETHERLANDS}

doi:10.1136/jech.2011.142976j.79

${ }^{1} \mathrm{~A}$ Pijpe, ${ }^{*}{ }^{2} \mathrm{R}$ Vermeulen, ${ }^{2} \mathrm{P}$ Slottje, ${ }^{1} \mathrm{~F}$ van Leeuwen, ${ }^{1} \mathrm{M}$ Rookus. ${ }^{1}$ Netherlands Cancer Institute, Amsterdam, The Netherlands; ${ }^{2}$ Institute for Risk Assessment Sciences, Utrecht, The Netherlands

A new prospective cohort study (the Nightingale Study) among nurses in the Netherlands was initiated in collaboration with the Institute for Risk Assessment Sciences (IRAS, Utrecht University) and the nationwide register of healthcare professionals (BIG-register of the Ministry of Health, Welfare and Sport, The Hague). Exposure to light-at-night has been suggested as a contributing cause of breast cancer (IARC classification "probable human carcinogen, $2 A$ "). Since shift- and night-time work is prevalent and increasing in modern societies, this exposure may contribute to the continuing elevation in breast cancer risk and may be of public health concern. This study will provide insight into, among others, the potential association between occupational exposures (eg, shift work, electromagnetic fields) and the risk of cancer and other diseases, and on potential biological mechanisms. The total projected study population is 50000 women. Through the BIG-register, women with a nurse diploma and who are younger than 60 years, will be asked to complete a (web-based or paper) questionnaire, sign an informed consent, and donate toenails for DNA analyses (eg, clock genes). In the spring of 2011, a pilot study $(\mathrm{N}=800)$ will be conducted of which the results will be presented. Specific attention will be directed to potential shift-work related selection.

\section{P2-247 IMPACT OF RISK FACTORS FOR NON-FATAL ACUTE MYOCARDIAL INFARCTIONS AMONG SRI LANKANS}

doi:10.1136/jech.2011.142976j.80

J Pinidiyapathirage, ${ }^{*}$ A R Wickremasinghe. Depatment of Public Health, University of Kelaniya, Ragama, Sri Lanka

Introduction The impact of risk factors for acute myocardial infarctions (AMI) differs across populations. The aim of this study was to determine the impact of established risk factors for the occurrence of 